\title{
Breaking Bad News - A Psychological Approach
}

\author{
*Mrs.Prabavathy.S
}

\section{Abstract:}

Breaking bad news is if we do it badly, the patients are family members may never forgive us; if we do it well, they may never forget us. (Buckman, 1992)1. ASCO survey identified several barriers to break bad news such as how to be honest with the patient and not destroy hope (55\%), cannot deal with the patient's emotions (25\%) and not find the right amount of time(10\%). ${ }^{2}$ Psychological Approaches of Breaking Bad News are ABCDE approach, SPIKES approach, SAAIQ Emergency approach, IJPC approach and SAD NEWS approach ${ }^{3}$. SPIKES PROTOCOL Psychological approaches are the targets being done to correct the faulty behaviors and habits, along with damaging words, thoughts, interpretation and feedback that direct strategies for the daily living 4. And it is often practiced by Psychiatrist, Psychiatric Nurse clinical psychologist, social workers and counselors. Breaking bad news in Psychological approach is a sensitive part of the health team member including physicians and Nurses need to be strong in effective therapeutic communication during the crisis situation ${ }^{5}$.

\section{Keywords: Breaking Bad news and Psychological Approach}

\section{INTRODUCTION}

Breaking bad news is ifwe do it badly, the patients are family members may never forgive us; if we do it well, they may never forget us. (Buckman, 1992) .Information that negatively alters the patient's view of the future, providing the current critical situation of patient information to the relatives and exploring the crisis situation.Someof the examples of Bad News includes Life threatening illnesses(E.g. Cancer, HIV), Degenerative conditions (E.g. Alzheimer/dementia.), Chronic illnesses (E.g. Rheumatoid arthritis /systemic lupus erythematosus), Mental illnesses (E.g. Schizophrenia/ BPAD). ${ }^{1}$

\section{WHAT ARE THE BARRIERS TO BREAKING BAD NEWS?}

ASCO survey identified several barriers to break bad news such as how to be honest with the patient and not destroy hope (55\%), cannot deal with the patient's emotions (25\%) and not find the right amount of time(10\%). ${ }^{2}$

\section{WHY BREAKING BAD NEWS IS NEEDED?}

It forms a part of clinical practice. Non disclosure is no longer considered ethical. It helps to gain better psychological adjustment by patients. Breaking Bad News facilitates open discussion among patient, relatives and doctors. Empowers patient by allowing them a 
Greater say in treatment ${ }^{2}$

\section{Psychological Approaches of Breaking Bad News $^{3}$}

- Rabow and McPhee's - ABCDE approach

- Baile and Buckman - SPIKES approach

- SAAIQ - Emergency approach

- BREAKS approach by IJPC

- SAD NEWS approach - Q.U /Canada

\section{“ABCDE” APPROACH}

1. Advanced preparation

2. Build therapeutic environment

3. Communicate well

4. Deal with patient and family reaction

5. Encourage and validate emotions

\section{Advanced preparation}

Advanced preparation includes what the patients already know? It is essential to arrange for the presence of a support person and appropriate family. Time and place selected to be undisturbed. Prepare yourself emotionally and decide is going to be told oneself

\section{Build therapeutic environment}

Arranging a private, quiet place without interruptionsand providing adequate seating for all is essential.Sitting close enough to touch if appropriate.Reassure about pain, suffering, abandonment

\section{Communicate well}

Be direct - "I am sorry that I have bad news for you". Therapeutic Communication Techniques plays a vital role.Allow for silence and use touch appropriate.

\section{Deal with patient and family reaction.}

Assessing patient reaction: physiologic responses, cognitive coping strategies, affective responses and Listen actively, explore, have empathy.

\section{Encourage and validate emotions}

Address further needs: What is the patient's immediate and near-term Suicidal Plan? Makingappropriate referral for more support.Explore what the news means to the patient and expressing your own feelings. ${ }^{3}$

\section{SPIKES PROTOCOL}

Step 1: Setting

STEP 2: Perception

STEP 3: Invitation

STEP 4: Knowledge

STEP 5: Emotion

STEP 6: Strategy and Summary

\section{Setting up in privacy}

Privacy is provided to patient and determination of who else should be present along with them is done. Introduce self and ensure that there are no interruptions. Provide comfortable space and welcoming environment

\section{Perceptions of the patients}

Prepare yourself before speaking and ask about patient's perception of what is going on.

\section{Invitation to break news}

Ask questions to invite the patient into conversation and ask how much information the patient wants to hear

\section{Knowledge}

Deliver the message.

Using of plain language and be mindful of body language helps in getting to the point easier. Information to be given in small chunks and give a pause to check for the reaction Use "teach back" to verify the message was received...

\section{Emotions}

Always to be prepared for patient's and family's emotional response after breaking of bad news. Anticipate fear, anger, sadness, denial, guilt and to be 
conscious of your own response. Provide comfort to the patient.

\section{Strategy}

Assess the patient readiness for planning and negotiate next step. Verify support structureknowledge and answer questions. Using of "teach back" technique and follow up

\section{"SAAIQ" APPROACH}

$>$ SET the sense as soon as possible.

$>$ ASSESS the understanding of the attendant.

$>$ ALERT them that I have bad news.

$>$ INFORM in clear, understandable words.

$>$ QUICKLY repeat summary of the situation

\section{“BREAKS" APPROACH}

$>$ Background

$>$ Rapport

$>$ Explore

$>$ Announce

$>$ Kindling

$>$ Summaries

\section{“SAD NEWS" Approach}

- Set up \& sit down

- Ask,don't tell

- Deliver the news

- No fancy lingo

- Expect, permit \& response to emotion

- Wait

- Support and summarize

\section{What to do?}

Always comfort the patient and provide privacy. It is essential to determine what the patient already knows and warn the patient that bad news is coming. Break the Bad News and identify the patient's main concern to support. Summarize and check understanding of the patient to offer realistic hope. Arrange follow up and make sure that someone is with the patient when he leaves. ${ }^{4}$

\section{What notto do?}

Do not hurryand give all the information in one go.It is essential not to give too much information. Avoid medical jargon or unclear language/words. Do not lie or be economical with the truth be blunt. Words can be like loaded pistols/guns.Do not guess the prognosis ${ }^{5}$

\section{Example of breaking Bad News in Psychological approach}

WRONG WAY: "Your mother has had a severe IC bleed. She is in the ICU and has been intubated and ventilated. Neurosurgery has placed a ventriculostomy to reduce the pressure in her brain. We do not anticipate a good prognosis"

RIGHT WAY: "Your mother has had a severe Stroke. She is in the Intensive Care Unit and has been placed on life support. The brain surgeon has inserted a tube to reduce the pressure in her brain. We do not think that she will survive" we trust the God. ${ }^{6}$

\section{Psychological Approaches in Breaking the Bad News}

Psychological approaches are the targets being done to correct the faulty behaviors and habits, along with damaging words, thoughts, interpretation and feedback that direct strategies for the daily living. And it is often practiced by Psychiatrist, Psychiatric Nurse clinical psychologist, social workers and counselors. ${ }^{7}$

\section{TYPES OF PSYCHO THERAPEUTIC APPROACHES}

- Psychodynamic therapy

- Behavioral therapy

- Cognitive therapy

- Essential / humanistic therapy

- Diversion therapy

Skills Adopted In Breaking the Bad News

- Preparing yourself to feel badly

- Set the context

- Deliver the bad news clearly and unequivocally 
- Ask for questions

- Never destroy hope

- Express your commitment of support

- Make a plan

- Follow up

\section{CONCLUSION:}

Breaking bad news is a complex yet important communication skill that all Health professionals must be able to execute well. There are many benefits of breaking bad news, not only for the patient, but also for the medical team managing the patient. It empowers and informs the patient and allows them to plan ahead. Breaking bad news in Psychological approach is a sensitive part of the health team member including physicians and Nurses need to be strong in effective therapeutic communication during the crisis situation.

\section{REFERENCES:}

1. Booth $\mathrm{K}$ et al (1996) Perceived Professional Support and the use of Blocking Behaviours by HospiceNurses. Journal of Advanced Nursing,24, 522527

2. Buckman R (1992) Breaking Bad News: A Guide for Health Care Professionals. Baltimore: Johns Hopkins University Press.

3. Dickson D, Hargie O Morrow N (1997) Communication Skill Training for Health Professionals. 2nd Edition Publishers Chapman and Hall.

4. Stewart MA (1996). Effective communication and health outcomes: a review;

5. Canadian Medical Association Journal, 152:1423-1438

6. Ptacek JT, Eberhardt TL. Breaking bad news.A review of the literature.JAMA 1996;276(6):496-502.

\section{ACUTE RESPIRATORY DISTRESS SYNDROME}

(묘묭)

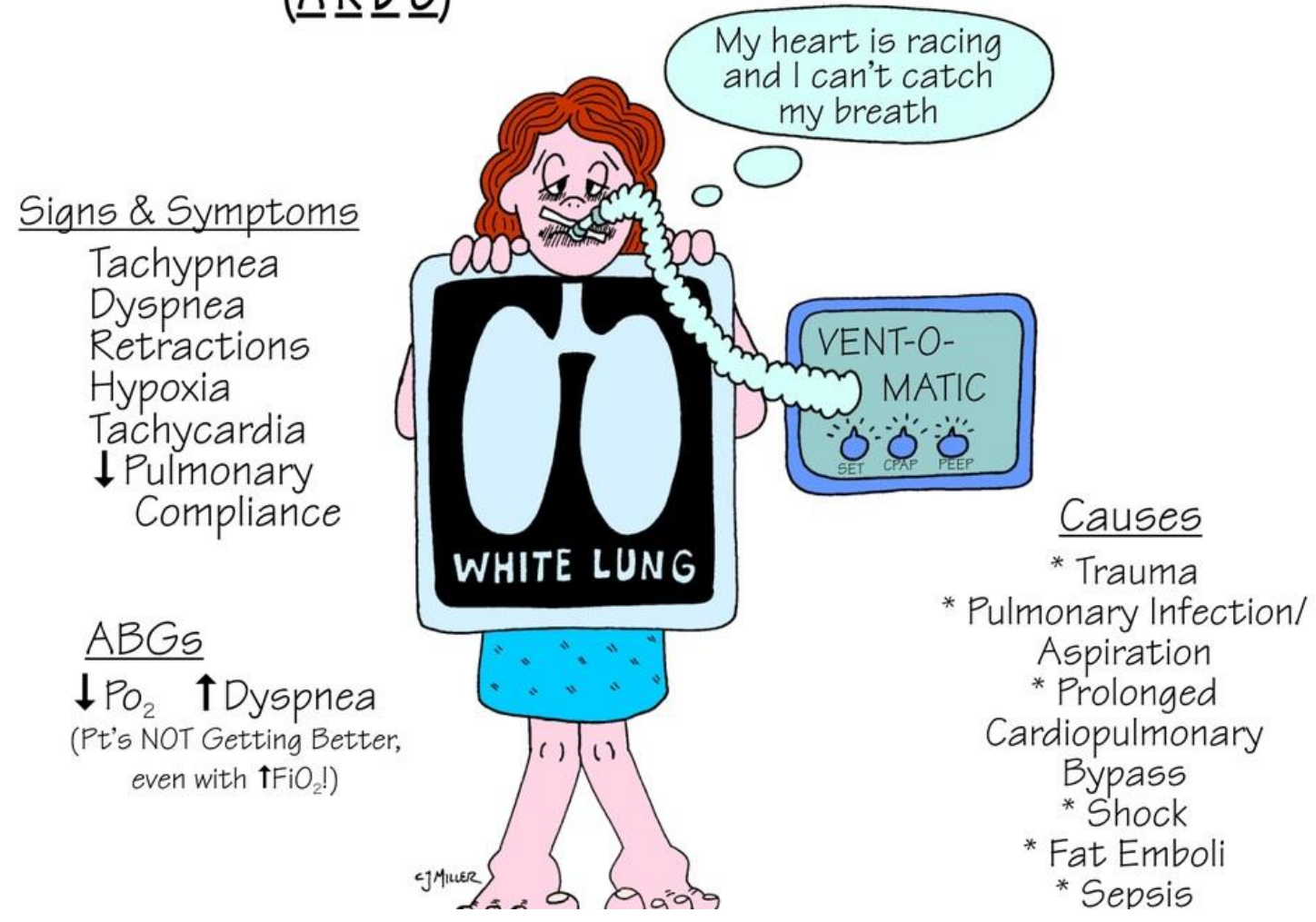

